\title{
$R G R$ variants in different forms of retinal diseases: The undetermined role of truncation mutations
}

\author{
JIALI LI, XUESHAN XIAO, SHIQIANG LI, XIAOYUN JIA, XIANGMING GUO and QINGJIONG ZHANG \\ State Key Laboratory of Ophthalmology, Zhongshan Ophthalmic Center, \\ Sun Yat-sen University, Guangzhou, Guangdong 510060, P.R. China
}

Received October 8, 2015; Accepted September 7, 2016

DOI: $10.3892 / \mathrm{mmr} .2016 .5847$

\begin{abstract}
It has been previously reported that mutations in retinal $\mathrm{G}$ protein coupled receptor $(R G R)$ are associated with retinitis pigmentosa. The present study aims to systemically analyze the potential role of variants of $R G R$ in retinal diseases. Variants in coding regions and splice sites of $R G R$ were selected from a whole exome sequencing dataset of 820 probands with various forms of genetic ocular diseases. Potential variants of $R G R$ were further confirmed by Sanger sequencing and analyzed in available family members. Clinical data was reviewed for patients with $R G R$ variants. As a result, a total of five variants in $R G R$ were detected in six probands with different types of ocular diseases. Of the five variants, two were novel heterozygous truncation variations, c.266C>A (p.S89*) and c.722_723delCC (p.S241Yfs*29), identified in two probands with high myopia and confirmed by Sanger sequencing. Segregation analysis on available family members demonstrated p.S89* and p.S241Yfs 29 were also present in unaffected relatives. The other three variants of $R G R$ were heterozygous missense variants randomly occurring in patients with different genetic ocular diseases. No homozygous or compound heterozygous variants were detected. The results of the present study suggested that the heterozygous truncation variants in $R G R$ were less likely to be pathogenic. Further studies are expected to evaluate the pathogenicity of variants in $R G R$.
\end{abstract}

\section{Introduction}

Retinal G protein coupled receptor $(R G R)$ [Online Mendelian Inheritance in Man (MIM) 600342)] encodes a putative retinal G-protein coupled receptor, a rhodopsin homologue, expressed

Correspondence to: Professor Qingjiong Zhang, State Key Laboratory of Ophthalmology, Zhongshan Ophthalmic Center, Sun Yat-sen University, 54 Xianlie Road, Guangzhou, Guangdong 510060, P.R. China

E-mail: zhangqji@mail.sysu.edu.cn

Key words: RGR, mutation, retinitis pigmentosa, high myopia, whole exome sequencing exclusively in the retina (1-3). $R G R$ is essential for the visual cycle as it is involved in the production of 11-cis-retinal (4). An abnormal visual cycle affects visual perception and ultimately leads to ocular disorders (5). However, the association of $R G R$ with specific ocular diseases has been rarely reported. Only a homozygous missense mutation and a heterozygous frameshift mutation have been reported to be associated with retinitis pigmentosa and choroidal sclerosis, respectively (5). However, the involvement of $R G R$ in the pathogenesis of retinitis pigmentosa has not been implicated in subsequent studies $(6,7)$. The potential role of $R G R$ in retinal diseases remains to be elucidated. Thus, the present study aims to systemically evaluate and analyze the potential role and pathogenicity of variants in $R G R$. This will be done with reference to a whole exome sequencing dataset from 820 probands with different forms of genetic ocular diseases.

\section{Materials and methods}

Patients. The present study is part of a project to investigate genetic defects associated with genetic ocular diseases using whole exome sequencing. Whole exome sequencing was performed on samples from 820 probands with different forms of genetic ocular diseases. All patients were recruited from the clinic of the Zhongshan Ophthalmic Center (Guangzhou, China). Written informed consent was obtained from the participants or their guardians, following the tenets of the Declaration of Helsinki. The present study was approved by the Institutional Review Board of Zhongshan Ophthalmic Center.

Sequencing. Whole exome sequencing was performed using a SureSelect Human All Exon Enrichment kit V4 (Agilent Technologies, Inc., Santa Clara, CA, USA) or TruSeq Exome Enrichment Kit (Illumina, Inc., San Diego, CA, USA) as previously described $(8,9)$. Variants in coding regions and splice sites in $R G R$ were selected from the whole exome sequencing data of 820 probands with various genetic ocular diseases. Those variants with minor allele frequency (MAF) $\leq 0.01$ were further analyzed by functional prediction using online methods, including SIFT (sift.jcvi.org/www/SIFT_enst_submit.html) (10), PolyPhen-2 (genetics.bwh.harvard.edu/pph2/) (11), and Berkeley Drosophila Genome Project (www.fruitfly.org/) (12). The MAF of each variant was obtained from the public databases, 
Table I. Primers used for amplification and sequencing of RGR.

\begin{tabular}{lcccc}
\hline Primer & \multicolumn{1}{c}{$\begin{array}{c}\text { Forward primer } \\
\left(5^{\prime}-3^{\prime}\right)\end{array}$} & $\begin{array}{c}\text { Reverse primer } \\
\left(5^{\prime}-3^{\prime}\right)\end{array}$ & $\begin{array}{c}\text { Amplicon } \\
(\mathrm{bp})\end{array}$ & $\begin{array}{c}\text { Annealing } \\
\text { temperature }\left({ }^{\circ} \mathrm{C}\right)\end{array}$ \\
\hline RGR-86008695 & GCAGCATTCAGGAACACACA & CCCTGCCTCTTATCCTCTCC & 283 & $65-58^{\mathrm{a}}$ \\
RGR-86017741 & TGCTGACCTGGTTTTCTTGG & AGGAAGAGACTGACACAGAGGT & 300 & $65-58^{\mathrm{a}}$ \\
\hline
\end{tabular}

${ }^{\mathrm{a}}$ Gradient annealing temperatures from 65 to $58^{\circ} \mathrm{C}$. RGR, retinal $\mathrm{G}$ protein coupled receptor.

Table II. Summary of variants in RGR detected in probands with different forms of genetic ocular diseases.

\begin{tabular}{|c|c|c|c|c|c|c|c|c|c|c|}
\hline \multirow[b]{2}{*}{ Gene } & \multirow[b]{2}{*}{ Chromosome } & \multirow[b]{2}{*}{ Position } & \multirow[b]{2}{*}{ Sample } & \multicolumn{2}{|c|}{ Variation } & \multirow[b]{2}{*}{ Status } & \multirow[b]{2}{*}{ SIFT } & \multirow{2}{*}{$\begin{array}{c}\text { Poly } \\
\text { Phen-2 }\end{array}$} & \multicolumn{2}{|c|}{ MAF } \\
\hline & & & & Nucleotide & Amino acid & & & & $1000 \mathrm{G}$ & EVS \\
\hline RGR & chr10 & 86007503 & HM345, QT371 & c. $236 \mathrm{G}>\mathrm{A}$ & p.R79H & Hetero & $\mathrm{D}$ & B & None & None \\
\hline RGR & $\operatorname{chr} 10$ & 86007377 & QT1072 & c. $110 \mathrm{C}>\mathrm{T}$ & p.T37I & Hetero & D & B & None & None \\
\hline RGR & $\operatorname{chr} 10$ & 86008695 & HM723 & c. $266 \mathrm{C}>\mathrm{A}$ & p.S89* & Hetero & - & - & None & None \\
\hline RGR & $\operatorname{chr} 10$ & 86012764 & QT90 & c. $522 \mathrm{C}>\mathrm{G}$ & p.D174E & Hetero & $\mathrm{T}$ & D & None & None \\
\hline RGR & $\operatorname{chr} 10$ & 86017741 & HM824 & c.722_723delCC & p.S241Yfs ${ }^{*} 29$ & Hetero & - & - & None & None \\
\hline
\end{tabular}

D, damaging; B, benign; T, tolerate; NA, not available/not applicable; EVS, Exome Variant Sever; MAF, minor allele frequency; RGR, retinal $\mathrm{G}$ protein coupled receptor; $1000 \mathrm{G}, 1000$ Genomes database.

A

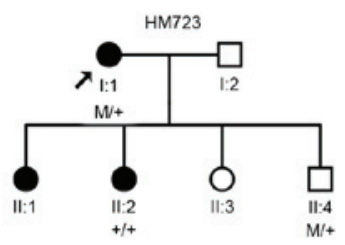

B
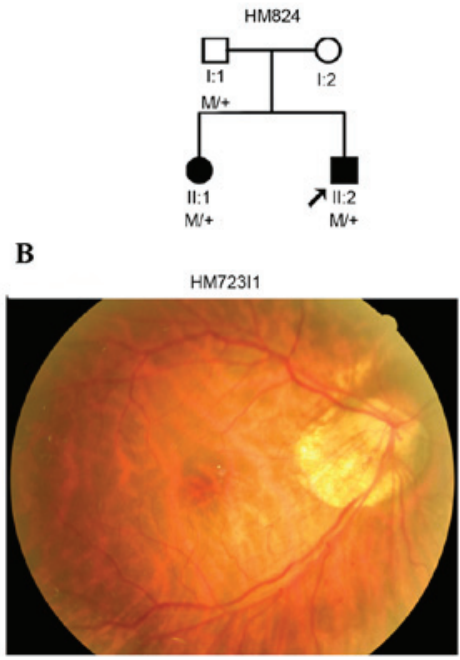

HM72311

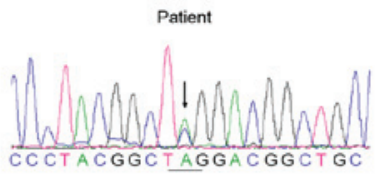

RGR c.268C >A

C
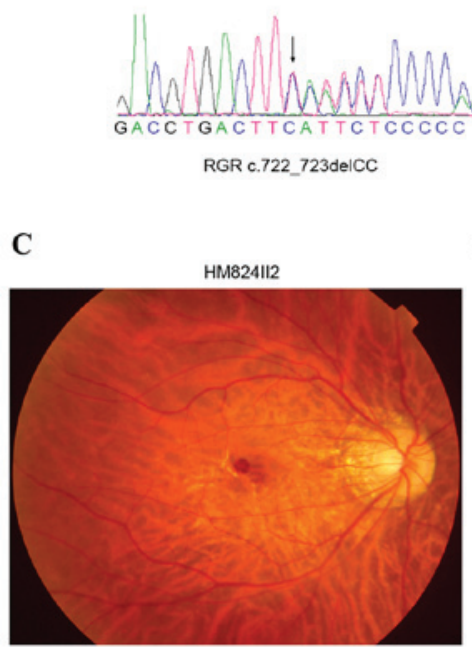

RGR C.722_723delCC
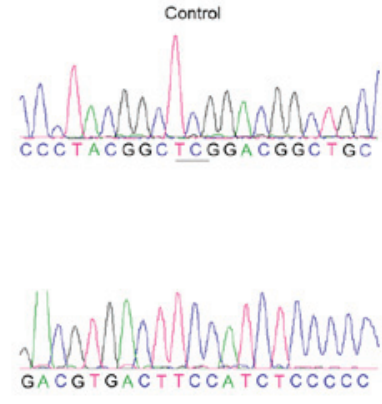

D

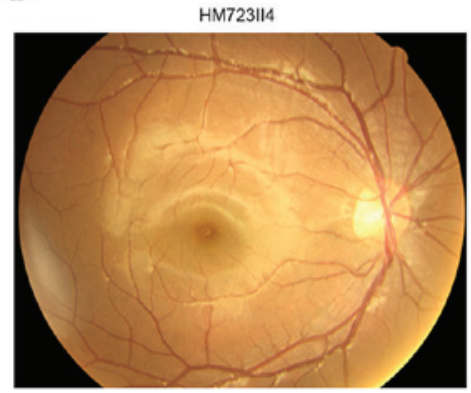

Figure 1. Truncation variants in retinal G protein coupled receptor identified in two probands with early-onset high myopia. (A) Sequence chromatography and pedigrees of HM723 and HM824. Sequence changes detected in the patients with early-onset high myopia were shown in the left column, whereas normal sequences were shown in the right column. Fundus photographs from right eyes of high myopes, (B) HM723I1 and (C) HM824II2, and (D) unaffected relative, HM723II4. M, mutation; +, wild-type.

dbSNP (www.ncbi.nlm.nih.gov/projects/SNP/), 1000 Genomes (www.1000genomes.org/), and the Exome Variation Server (evs.gs.washington.edu/EVS/). Potential variants of $R G R$ were further confirmed by Sanger sequencing and validated in available family members. Primers used for amplification of fragments were designed using the Primer3 online tool (bioinfo. 
ut.ee/primer3-0.4.0/) and are presented in Table I. The methods used for amplification, sequencing, and analysis of the target fragments were as previously described (13). The descriptions of the variants are consistent with the nomenclature for sequence variations (www.hgvs.org/mutnomen/) (14).

\section{Results}

Following a review of the whole exome dataset of 820 probands with different forms of genetic ocular diseases, a total of 5 variants of $R G R$ were detected in 6 of the 820 probands. Of the five variants, two were heterozygous truncation variants, c.266C $>$ A (p.S89*) and c.722_723delCC (p.S242Yfs"29), identified in two probands with early-onset high myopia (Fig. 1A and Table II). These two variants were further confirmed by Sanger sequencing (Fig. 1A). Segregation analysis on available family relatives identified that p.S89* and p.S242Yfs"2 did not co-segregate with high myopia, they were present in the unaffected relatives but absent in the affected relatives (Fig. 1A). The other three variants were heterozygous missense variants and identified in four probands, one with high myopia, one with cone-rod dystrophy, and two with Leber congenital amaurosis (Table II). No homozygous or compound heterozygous variants in $R G R$ were detected.

The two probands with $R G R$ truncation variants complained of poor vision at younger than primary school age, but denied photophobia and night blindness (Table III). Fundus examination demonstrated tigroid fundus and temporal crescent of optic nerve head (Fig. 1B and C), which was consistent with the diagnosis of high myopia. Neither marked retinal vessel attenuation nor bone corpuscle type of pigmentation were observed (Fig. 1B and C). However, additional family members with $R G R$ truncation variants (HM723II4 and HM824I1) were unaffected individuals without high myopia (Table III) and did not have any notable signs of abnormal fundus changes (Fig. 1D).

\section{Discussion}

Based on the whole exome sequencing dataset from 820 probands with different forms of genetic ocular diseases, two heterozygous truncation variants in $R G R$ were identified in two probands with high myopia, but these did not co-segregate with high myopia. The other three variants in $R G R$ were heterozygous missense variants, and occurred randomly in four patients with different forms of genetic ocular diseases. No homozygous or compound heterozygous variants were detected in $R G R$.

Only a limited number of $R G R$ variants have been previously reported (5-7). Among them, only two have been identified in two families with either retinitis pigmentosa or choroidal sclerosis (5), a homozygous c.196A >C (p.Ser66Arg) variant identified in a family with autosomal recessive retinitis pigmentosa and a heterozygous c.824dupG (p.M275Ifs ${ }^{*} 83$ ) insertion identified in a small family with autosomal dominant choroidal sclerosis (5). Subsequently, screening of $R G R$ in two independent studies only identified a number of less likely pathogenic variants and polymorphisms, as reviewed in Table IV. Of the five variants detected in the current study, two were heterozygous novel truncations, p.S89* and p.S242Yfs" 29 , which presented in two probands with high myopia. These two variants and the previously reported heterozygous variant, c.824dupG, were 
Table IV. Reported variants in RGR.

\begin{tabular}{|c|c|c|c|c|c|c|c|c|}
\hline First author, year & Nucleotide & Protein & Status & $\begin{array}{l}\text { MAF } \\
\text { case }\end{array}$ & $\begin{array}{l}\text { Phenotype } \\
\text { in case }\end{array}$ & $\begin{array}{l}\text { Co-segre } \\
\text { gation }\end{array}$ & $\begin{array}{l}\text { MAF in } \\
\text { control }\end{array}$ & Refs. \\
\hline Morimura, 1999 & c.824dupG ${ }^{\mathrm{a}}$ & Gly275Ilefs*83 & Hetero & $1 / 1684$ & $\operatorname{adRP}^{b}$ & Yes & $0 / 190$ & (5) \\
\hline Morimura, 1999 & c. $196 \mathrm{~A}>\mathrm{C}^{\mathrm{a}}$ & Ser66Arg & Homo & $2 / 1684$ & $\operatorname{arRP}$ & Yes & 0/190 & (5) \\
\hline Morimura, 1999 & IVS5-12A $\rightarrow \mathrm{G}^{\mathrm{c}}$ & Splicing & Hetero & $1 / 1684$ & sRP & NA & $0 / 190$ & (5) \\
\hline Morimura, 1999 & IVS6 $+3 \mathrm{~A} \rightarrow \mathrm{G}^{\mathrm{c}}$ & Splicing & Hetero & $1 / 1684$ & sRP & NA & 0/190 & (5) \\
\hline Morimura, 1999 & IVS6 $+5 \mathrm{~A} \rightarrow \mathrm{G}^{\mathrm{c}}$ & Splicing & Hetero & $4 / 1684$ & sRP & NA & $1 / 190$ & (5) \\
\hline Morimura, 1999 & $\mathrm{GTG} \rightarrow \mathrm{TTG}^{\mathrm{c}}$ & Val132Leu & Hetero & $1 / 1684$ & sRP & NA & 0/190 & (5) \\
\hline Morimura, 1999 & $\mathrm{CAC} \rightarrow \mathrm{AAC}^{\mathrm{c}}$ & His 152Asn & Hetero & $1 / 1684$ & sRP & NA & $0 / 190$ & (5) \\
\hline Morimura, 1999 & $\mathrm{GCA} \rightarrow \mathrm{ACA}^{\mathrm{c}}$ & Ala234Thr & Hetero & $1 / 1684$ & sRP & NA & $0 / 190$ & (5) \\
\hline Morimura, 1999 & $\mathrm{TCC} \rightarrow \mathrm{TTC}^{\mathrm{c}}$ & Ser241Phe & Hetero/Homo & $6 / 1684$ & adRP; sRP & NA & $1 / 190$ & (5) \\
\hline Bernal, 2003 & $\mathrm{TCC} \rightarrow \mathrm{TTC}^{\mathrm{c}}$ & Ser241Phe & Hetero & $10 / 184$ & $\operatorname{arRP}$ & No & $5 / 190$ & $(6)$ \\
\hline Bernal, 2003 & nt $615 \mathrm{G}>\mathrm{A}^{\mathrm{c}}$ & $\mathrm{p} .=$ & Hetero & $1 / 184$ & $\operatorname{arRP}$ & NA & NA & (6) \\
\hline Bernal, 2003 & IVS6 $+5 \mathrm{~A}>\mathrm{G}^{*_{\mathrm{c}}}$ & Splicing & Hetero & $1 / 184$ & $\operatorname{arRP}$ & No & $0 / 190$ & (6) \\
\hline Ksantini, 2010 & c. $466 C>\mathrm{A}^{\mathrm{c}}$ & His 156Asn & Hetero/Homo & $3 / 662$ & arRP; sRP & NA & $0 / 100$ & $(7)^{d}$ \\
\hline Ksantini, 2010 & c. $474 \mathrm{C}>\mathrm{T}^{\mathrm{c}}$ & $\mathrm{p} .=$ & NA & $1 / 184$ & sRP & NA & NA & (7) \\
\hline Morimura,1999; Bernal, 2003 & IVS5+16C $\rightarrow \mathrm{T}^{\mathrm{e}}$ & Intronic & NA & 0.07 & NA & NA & NA & $(5,6)$ \\
\hline Morimura, 1999; Bernal, 2003 & nt $19 \mathrm{C}>\mathrm{T}^{\mathrm{e}}$ & $\mathrm{p} .=$ & NA & 0.07 & NA & NA & NA & $(5,6)$ \\
\hline Morimura, 1999; Bernal, 2003 & nt $27 \mathrm{C}>\mathrm{T}^{\mathrm{e}}$ & $\mathrm{p} .=$ & NA & 0.47 & NA & NA & NA & $(5,6)$ \\
\hline Morimura, 1999; Bernal, 2003 & nt $459 \mathrm{C}>\mathrm{T}^{\mathrm{e}}$ & $\mathrm{p} .=$ & NA & 0.37 & NA & NA & NA & $(5,6)$ \\
\hline Ksantini, 2010 & c. $19 \mathrm{C}>\mathrm{T}^{\mathrm{e}}$ & $\mathrm{p} .=$ & NA & 0.03 & NA & NA & NA & (7) \\
\hline Ksantini, 2010 & c. $27 \mathrm{~T}>\mathrm{C}^{\mathrm{e}}$ & $\mathrm{p} .=$ & NA & 0.36 & NA & NA & NA & (7) \\
\hline Ksantini, 2010 & c. $-111 \mathrm{~A}>\mathrm{G}^{\mathrm{e}}$ & Non coding & NA & 0.72 & NA & NA & NA & (7) \\
\hline Ksantini, 2010 & c. $79+59 \mathrm{C}>\mathrm{T}^{\mathrm{e}}$ & Non coding & NA & 0.02 & NA & NA & NA & (7) \\
\hline Ksantini, 2010 & c. $642+16 \mathrm{G}>\mathrm{A}^{\mathrm{e}}$ & Non coding & NA & 0.07 & NA & NA & NA & (7) \\
\hline Ksantini, 2010 & c. $65 \mathrm{~A}>\mathrm{G}^{\mathrm{e}}$ & Non coding & NA & 0.11 & NA & NA & NA & (7) \\
\hline Ksantini, 2010 & c.. $^{*} 100 \_101$ insA $^{\mathrm{e}}$ & Non coding & NA & 0.06 & NA & NA & NA & (7) \\
\hline
\end{tabular}

${ }^{a}$ Mutations associated with RP; ${ }^{b}$ originally diagnosed with choroidal sclerosis; ' less likely to be pathogenic variants; ${ }^{\mathrm{d}}$ the variant was predicted to be damaging by Polyphen but not conserved among species; ${ }^{e}$ polymorphisms. MAF, minor allele frequency; RP, retinitis pigmentosa; adRP, autosomal dominant RP; arRP, autosomal recessive RP; sRP, sporadic RP; NA, not available; RGR, retinal G protein coupled receptor.

located in exon 3, exon 6 , and exon 7 of $R G R$, respectively, and have been predicted to result in an abnormal transcript. They were absent in the Exome Variants Server and 1000 Genomes databases. However, the p.S89* and p.S242Yfs" 29 variants were also detected in unaffected family members without any abnormalities of the fundus. Furthermore, searching of the Exome Variants Server and 1000 Genomes databases revealed a further five truncation variants of $R G R, \mathrm{c} .190 \mathrm{G}>\mathrm{A}$ (p.W47*) in $1 / 4406$ alleles, c.775del1 (p.M260Wfs" 43 ) in 99/12,518 alleles, c.775A>T (p.K259") in 2/13,006 alleles, c.796_797insCC (p.I267Pfs"37) in 1/12,518 alleles, and c.877C>T (p.R293*) in 1/13,006 alleles. These findings suggest that heterozygous truncation variants of $R G R$ are less likely to be pathogenic. Furthermore, it has been observed that heterozygous missense variants of $R G R$ have a similar distribution among probands with different forms of genetic ocular diseases and thus, may not be pathogenic. The pathogenicity of the homozygous or compound variants of $R G R$, remains to be elucidated, as no such variants were detected in the current study.

In conclusion, the results of the present study suggest that the potential role of heterozygous truncation of $R G R$ in ocular diseases remains to be determined. Additional studies are required to provide further understanding.

\section{Acknowledgements}

The present study was supported by the National Natural Science Foundation of China (grant no. U1201221), the Natural Science Foundation of Guangdong (grant no. S2013030012978), and the Fundamental Research Funds of the State Key Laboratory of Ophthalmology (grant no. 2012PI01).

\section{References}

1. Jiang M, Pandey S and Fong HK: An opsin homologue in the retina and pigment epithelium. Invest Ophthalmol Vis Sci 34: 3669-3678, 1993.

2. Shen D, Jiang M, Hao W, Tao L, Salazar M and Fong HK: A human opsin-related gene that encodes a retinaldehyde-binding protein. Biochemistry 33: 13117-13125, 1994.

3. Trifunovic D, Karali M, Camposampiero D, Ponzin D, Banfi S and Marigo V: A high-resolution RNA expression atlas of retinitis pigmentosa genes in human and mouse retinas. Invest Ophthalmol Vis Sci 49: 2330-2336, 2008. 
4. Chen P, Lee TD and Fong HK: Interaction of 11-cis-retinol dehydrogenase with the chromophore of retinal g protein-coupled receptor opsin. J Biol Chem 276: 21098-21104, 2001.

5. Morimura H, Saindelle-Ribeaudeau F, Berson EL and Dryja TP: Mutations in RGR, encoding a light-sensitive opsin homologue, in patients with retinitis pigmentosa. Nat Genet 23: 393-394, 1999.

6. Bernal S, Calaf M, Garcia-Hoyos M, Garcia-Sandoval B, Rosell J, Adan A, Ayuso C and Baiget M: Study of the involvement of the RGR, CRPB1, and CRB1 genes in the pathogenesis of autosomal recessive retinitis pigmentosa. J Med Genet 40: e89, 2003.

7. Ksantini M, Sénéchal A, Bocquet B, Meunier I, Brabet P and Hamel CP: Screening genes of the visual cycle RGR, RBP1 and RBP3 identifies rare sequence variations. Ophthalmic Genet 31: 200-204, 2010

8. Huang X, Li M, Guo X, Li S, Xiao X, Jia X, Liu X and Zhang Q Mutation analysis of seven known glaucoma-associated genes in Chinese patients with glaucoma. Invest Ophthalmol Vis Sci 55: 3594-3602, 2014
9. Li J, Jiang D, Xiao X, Li S, Jia X, Sun W, Guo X and Zhang Q: Evaluation of 12 myopia-associated genes in Chinese patients with high myopia. Invest Ophthalmol Vis Sci 56: 722-729, 2015.

10. Kumar P, Henikoff S and Ng PC: Predicting the effects of coding non-synonymous variants on protein function using the SIFT algorithm. Nat Protoc 4: 1073-1081, 2009.

11. Flanagan SE, Patch AM and Ellard S: Using SIFT and PolyPhen to predict loss-of-function and gain-of-function mutations. Genet Test Mol Biomarkers 14: 533-537, 2010.

12. Reese MG, Eeckman FH, Kulp D and Haussler D: Improved splice site detection in Genie. J Comput Biol 4: 311-323, 1997.

13. Jiang D, Li J, Xiao X, Li S, Jia X, Sun W, Guo X and Zhang Q: Detection of mutations in LRPAP1, CTSH, LEPREL1, ZNF644, SLC39A5, and SCO2 in 298 families with early-onset high myopia by exome sequencing. Invest Ophthalmol Vis Sci 56: 339-345, 2014.

14. den Dunnen JT and Antonarakis SE: Mutation nomenclature extensions and suggestions to describe complex mutations: A discussion. Hum Mutat 15: 7-12, 2000. 